\title{
Digital subjects: an introduction
}

\author{
Olga Goriunova ${ }^{1}$
}

Published online: 18 December 2018

(C) Springer Nature Limited 2018

What would it mean, to think that there are digital subjects? Not human subjects that are becoming more and more digitally mediated, but subjects formed in the computational ensembles out of data, models, and various other analytical operations? Such digital subjects could be nested set of abstractions assembled by algorithms, a dynamic data aggregate feeding upon logs of movement in space and time or on analyses of experiential presence in the networks. These could be subjects of clickstreams and cookies, of samples, templates, profiles, logs of phones' MAC addresses, and other things, produced by matching, scoring, probability estimations and predictions, - which act as novel configurations that arise from an unprecedented proliferation of techniques and infrastructures for data generation and analysis.

To think about such digital subjects would mean to ask many questions. How are they aggregated? What are the logics of their construction? What are their forms of unity and disunity? Are they even subjects? The subject is placed in complex power relations: subjects usually have will and agency. How do digital subjects get to operate as enactive subjects towards certain ends? What is their power? What is their politics? Are they always quasi-subjects, mere dolls operated by a puppeteer (such as the principle of value extraction, cognitive capitalism, racism)? Or are they also dynamic multiplexing formations that acquire their own principles of establishment of reality? What do they actually do? What do they do to humans? Do they match humans or not and how do they relate to them?

Articles in this special issue take on the challenge of thinking digital subjects from a variety of positions, informed by cultural theory, philosophy, media theory and software studies, social theory and art. Goriunova examines the ways in which digital subjects and data modality more generally gets anchored and

Olga Goriunova

olga.goriunova@rhul.ac.uk

1 London, UK 
establishes its own reality, values and practices by relying on the body principle, by designating biologically-inspired forms of identity as "true" and then stitching other forms of abstractions to them. She specifically looks at technologies of facial biometrics. Parisi proposes the emergence of another subject-an alien subject of AI, a subject produced by multilogics, incomputability, and complexity in machine learning. Kolozova takes issue with the whole notion of the subject, which, for her, is always ruptured between the body (the real) and the cognitive, semiotic machine. The rupture that Kolozova criticises seems to align with the seams of data worlds stitching together the geometry of the face and the experiences of subjectivity that Goriunova examines. Wark reformulates the proposition of the digital subject in relation to the notions of the user, grammars of action, technical entities and digital milieus, and proposes that digital subjects individuate in circulation, obtaining a regulatory capacity in relation to the humans. While people act within the available grammars of action as users, digital subjects scale, complexify and circulate, individuating themselves-and persons. Skeggs and Yuill suggest that networked capitalism (or Facebook in their case study) simultaneously aggregates, evaluates a digital subject and connects it to the bourgeois subject who can underwrite financial obligations. Both serve as sources of value that, in case of digital subjects, is wholly expropriated by Facebook.

All articles in the issue take up distinct conceptual problems that stem from the invitation to entertain the idea of the digital subject. The reader will not therefore find here detailed explications of effects of digitality on subjectivity; this is not a collection of works on the digital self or datafied personhood. The questions explored concern what it would mean to call digital subjects "subjects": are they productive of new ontological and epistemological demands? What are their truths? Even if digital subjects are entirely generated by self-sufficient computational machines or in response to novel forms of exploitation, their forms of emergence, coherence, and action are novel and underexplored.

A digital subject, therefore, is a subject of confusion: it can generally mean many, often contradictory, things. In terms of legal vocabulary of data protection acts, a related term, data subject, means an individual that a piece of personal data relates to. Here, the subject is a human being, who is pointed at, or irrevocably linked to by certain procedures, by their data. This is how the magazine Wired describes this link: “a 'join' between the Facebook user ID (that's you) and this outside third-party who knows what you bought, browsed, or who you voted for (probably). That join is permanent, irrevocable, and will follow you to every screen where you've used Facebook" (Garcia Martinez 2018). It is clear from this example, however, that it is not the human being per se who is the subject of their data. It is instead an abstraction of themselves (a user ID in this case) that acts as their data subject, standing in for the person (Goriunova 2019). This subject is repeatedly linked to data. This data subject is also linked to the living person through another set of procedures, which have specific socio-political, scientific, legal, psychological and techno-cultural genealogies. Such data subjects, found somewhere in-between human beings and data products of various kind, can be imagined as a weaver's shuttle that goes back and forth in perpetual movement, layering threads, one on top of the other, and in various directions, so that 
the data subject itself appears to be a multilayered multi-dimensional tangle of threads weaving persons and various data formations together.

Such subjects are found outside of subjectivity, as a subject position, or an abstraction. There are a rich variety of traditions of understanding the production of variant or previous instantiations of such subjects. The Foucauldian tradition offers one powerful approach. Especially in Foucault's early work, the subject is produced through objectification: of the speaking or labouring human being, or of a process of being alive, and through dividing, normalising practices (Foucault 1982). Foucault also considers self-production into a subject and the relationship between such subject and subjectivity, the experience of the living self, in the lectures at the College de France in his final years (Foucault 2005). The dynamic relationship of moving outside of oneself and coming back to a self through the care of oneself positions the notion of a subject as somewhat outside the self, where the subject is fragmented through the techniques of memory and reflection, or statistical technologies and other means and media. Here, self and subject are distinct and support each other in dynamic mutual production. Data and its various constructions, such as samples, templates, profiles, and others could then be considered as further traces and calculations of the subject that in turn remain in a dynamic relationship to the self. Data subjects are thus further abstractions of self, building, in new ways, on the previous forms of production of the self-subject dynamic.

Scholars working in the Foucauldian tradition, for instance, legal scholar Antoinette Rouvroy, criticise data analytical frameworks. Rouvroy argues that "the end of theory" proclaimed by the arrival of big data (Anderson 2008) disassembles a certain regime of objectivity previously constructed and maintained by science (with, for instance, hypothesis testing, laboratory trials, etc.) (Rouvroy 2013). Big data, according to her, does not perform material inscription, because it lacks historicity and is optimised for sales rather than to reveal truth. The points of bifurcation, at which the processual production of self, work on a subjectivity can happen are eliminated, by the design of the system, a priori. A substantial amount of critique of data analytics follows such-like characterisations, which are not without merit, but tend to depict digital infrastructures that data or algorithms are part of, as monolithic regimes, able to sustain internal agreement across a multitude of instruments, voices, models, practices, forms, scales and materials and able to envelop the human fully, without excess or tears in their smooth fabric of operation. Instead, one of the threads running through this special issue recognises the disagreement within digital "regimes" and the cacophony of various actors within it making do with partial, borrowed or adapted methods of work. Digital infrastructures work and don't work; digital systems create totalities that have limits; multiplexing operations of abstraction and computation gain and lose grip on lived reality. While it's important to recognise technology's totalising tendencies, those are not the only ones and by focusing exclusively on what is bad or doesn't yet work and thus failing to see the germination of new forms, critique itself may lose grip on the future.

Marxist analyses offer another, related and rich array of ways of understanding the construction of subjects. In this group of traditions, there is a call of modernity, or the hailing of ideology, or a capitalist mode of production and its determination of value that the individuals responds to, and that transforms them into a subject. 
Althusser has famously written about the "Hey, you there!" hail that makes the human "turn back" and respond, thereby fashioning themselves into the unit of a subject, constructed in the specific terms of the hail (Althusser 1970). In this tradition, Moulier-Boutang, for instance, argues that if labour-power was core to the industrial economy, it is the collective intelligence and inventiveness that is core to cognitive capitalism (Moulier-Boutang 2012). If it is the human capacity for thinking, emotion, communication that is at the core, then there is a similar process of abstraction of the human going on, to make sure a continuous measure of commodities is taken. Similarly to abstract labour, which is the measure of all things in capitalism, the abstraction of the human is the measure of the new value. Superseding and building on the Althusserian analysis of the reproduction of labour power through ideology, Moulier-Boutang argues that it is intelligence and communication, emotion, and all others capacities of the living human in a capitalist society that are at the heart of a new mode of production, and which are abstracted to be captured as "positive externalities", new sources of value (externalities are spill-overs of financial transactions that make an impact while remaining outside of economic considerations). These externalities, operating as financial derivatives, become subjects, relating to the future (Moulier-Boutang 2012,16) in their "response to [the] growing complexity and globalisation of interpretation processes [in the financialisation of the economy]" (Moulier-Boutang 2012, 16, 30).

And anyway, to use the term "subject" is at best ambivalent. Feminist theories, postcolonial studies, work in ecological humanities have shown how historically the subject was constructed as an epicentre of a conscience (at the exclusion of animal-like women hardly possessing a soul), reason and will (at the exclusion of many: women, people of colour, those who are disabled), intelligence and emotion (at the exclusion of many complex life forms). The subject is indivisible from various forms of repression, integral to its establishment. "Subject" as a term also plays into the hands of contemporary forms of extreme individualisation, all the while the coherency of the subject is an illusion. Subjects are enmeshed and generated in relationships: Braidotti's "nomadic subjects", for instance, propose a logic of decentralisation in subjectification that is transversal and relational (Braidotti 1994). Further work, focusing on affect (Blackman 2012), and contingency and plasticity as core to modern subjects (Valiaho 2014), in various ways emphasise subjectification as an open-ended relational process.

Digital subjects are not persons but are produced in relation to, among other things, individuals. Though living beings are only addressed as users in platforms such as Facebook, the value of the user comes from their subjectivity. The discussions above have not so far focused on subjectivity, while data subjects are arguably a narrower category than the digital subjects, the theme of this special issue. The situation is also complicated by an understanding that subjectivity encompasses within its generative processes various technologies: of coming outside of oneself and working on oneself, of memory, of performance, of vision, and so on. Subjectivity is something radically mediated; indeed, media forms have been claimed as technologies of subjectification by McLuhan, Foucault, Kittler and Stiegler, among others (McLuhan 1964; Foucault 2005; Kittler 1990; Stiegler 1998). Foucault and Kittler in particular focused on the personal journal, a 
familiar technique of making oneself into an individual, a subject endowed with a reflexivity or a soul, capable of self-improvement, and of picturing their own life, through this mediatory technique, as a coherent whole that makes sense (Foucault 2005; Kittler 1990). Making sense does not mean discovering hidden truths, but creating meaning through reflection, memory and the practices of assigning meaningfulness to events and experiences.

For Kittler, intent on eliminating the illusion of the subject, subjectivity equals a capacity for reflexive operationality and becomes redundant once self-guiding missiles and other machines using feedback and which are capable of self-adjustment in terms of speed, precision or complexity are produced. Machine subjects then inevitably outperform the human, conscribed to the role of a bio-unit in a global network, whose role is to supply the capacity of their nervous system to respond to stimuli in recursive loops (Halpern 2014). All that is left are nervous responses producing subjectivities outside subjectivities.

In this context, Haraway's redescription of the cyborg in a reclamation of the war machine still remains a radical and useful proposition (Haraway 1984). Guattari's transversal subjectivities rooted in sensibility, intelligence, sociability and culture, and which are produced in the new ethico-aesthetic paradigms that exclude neither technology nor ecology, offer another route for thought and action (Guattari 1989). Whereas journal writing was aligned with the metaphysical truth, today the question is not about the true self. The encounter is not between a sovereign self and computational infrastructures: in the ethos of joint becoming, a profound human-obsessed anxiety is generated that concerns the fates of subjectivities and people. What is the ontological status of this digital subject? Is it an artificial person? A mere representation? A collection of images? The digital subject doesn't rely on internalisation and doesn't necessarily draw the human into itself, making them an actor or a beneficiary of its construction, yet it still feeds on human (as well as non-human) capacities, attributes, and actions. The digital subject is a mutable construction that is always in the process of being assembled, and at the same time, it has a capacity to acquire enough consistency to become active, transducing various actions on persons back to them. Its ontological uniqueness perhaps lies in a way of capturing something in humans, and in coming back to them, through encounters, events, and processes of mobilisation. If the digital subject is produced within computational infrastructures that inscribe within themselves degrees of plasticity, what is it capable of, as an outside to the self, acting on the self? If its ontology is one of making, of epistemology, what are the relations between knowledge practices that make it up and the environments within which it constantly evolves and acts? What is foundational to it: mathematical logic, philosophical groundings of computer science, concrete practices, the capitalist search for value, assumptions, errors, episodes of violence, metaphors, or fictions? What are its ecologies: apparatuses of power, of subjectification, of knowledge, of automated reason?

There are many more questions posed here that cannot be answered within the space of one special issue: these are meant to indicate possible further directions of thinking about digital subjects. Some starting points are presented below. 


\section{A guide through contributions}

1. Goriunova's article explores some of the ontological promises of data infrastructures: one of these is a promise of accessing and capturing a unique, biologically grounded identity, which is then linked to the authentic subjectivity of a living person with the same promise of biological concreteness. Uniqueness based on the body principle is employed in the technology of biometric identification, and facial biometrics is a key case study used in this article. Goriunova closely reads the steps involved in biometric sampling and identification to show that the unique, universal and permanent identity of modernity is here never delivered. Uniqueness, instead, relies on a similarity expressed as a probability. Universality is nothing but a frequency of occurrence. Permanence is rather a measure of the capacity for variation that a profile can accommodate. Constructed out of adjustable parameters, weighted differently for different purposes, this identity is not located within a person, but is instead a process of linking the biological and the computational across people, datasets, and modelling methods. Such identity is a processual dynamic of a technical practice, and is assigned through epistemological means.

These peculiarly constructed claims to truth made in the mode of data have a partially familiar genealogy: as mentioned above, it has been argued that persons respond to being "hailed" by algorithms because they have been trained to fashion themselves into individuals in response to the general "hail" of modernity. But there are also new elements in these systems that now include within themselves a degree of openness and mutability. Goriunova argues that it is the anchoring in the biological principle that signals the attempts, on behalf of this data modality, to designate "the reality" and assert the values of its practices. Speculative data infrastructures, geared to calculate probabilities, she argues, assert truths based on the promise of physical indexicality in order to ground and layer further abstractions that in this way gain validity. The biological "truth" is hereby linked to all kinds of things claimed as truths; the processing of "life itself" becomes the processing of the world.

Starting with a short introduction, Goriunova first reflects on the face as, on one hand, a distributed site of multiplicity offering playfulness and disidentification (selfie culture, online personas), and on the other hand, absolute identification (it acts as an extension of the documentary tradition). She then examines the changing notions of identity and subjectivity in the computational data context, where biological facts are carefully interlinked with the rich experiences of subjectivities. After an analysis of facial biometrics, Goriunova focuses on "soft biometrics",- - all kinds of inscriptions, mobilisations and operationalisations layered on top of the "body principle",- - such as norms of beauty, brands, cats or the shadows of lampposts. As the capturing of the world expands beyond the human, it is in the politics of Earth itself that data worlds are stitched together in multiplexed, hyperdimensional variation.

2. There is no subject outside mediation and outside technology, and the myth of unitary subject of reason and will is dispelled, most recently, by how 
computational infrastructures parse the personal into the granular, cashing on its attributes. Taking this as her starting point, in her contribution to this special issue, Parisi asks what it means to think the subject of technology. What does it mean, for technology to have a subject?

Coming in the footsteps of the human subjects produced through "machinic enslavement", generated in relation to the assembly line and measured through labour, and following the subjects of "social subjection", that is, users enjoying an imaginary unity of self by orchestrating snippets of their datafied attributes, there is a third kind of subject, an alien subject, whose emergence, she argues, is linked to artificial intelligence, prediction and a "re-patterning of the space of thinking". Parisi offers a contemplation of a new mode of thought, which does not extend the project of Western metaphysics that reached its limit following the de-linking of truth and proof. Alien logic of the subject of technology does not inherit from the metaphysical crises of deductive reasoning and self-determination (here, the failure of deductive reason and of the colonising project of emancipation based on slavery are intrinsically conjoined in the same crisis of truth). Learning in machines, instead, articulates machine thinking outside of the servo-mechanistic model, in which machines are believed to be always modelled on and serve the human, and proposes $s$ it as novel and nuanced space of reasoning, housing a different, non selfpositing form of subject, with new political possibilities.

Parisi structures her argument as follows. She first examines two polar opposite sides of the debate on the effects of computation on the human: the "cybernetic hypothesis" and a re-purposing of technology to emancipate 'the human (here, the "accelerationist hypothesis"). According to the former, within cybernetic order, individual subjects are transformed into dynamic datasets, managed according to the shifting scenarios of probability. The illusion of the unity of the subject is achieved through seamless interactivity, and the only strategy of resistance is to obscure and obfuscate the transparent network. The latter, the proposition of accelerationism, which echoes, to some extent, Negri's work on networked capital, suggests a repurposing of instrumentality to reclaim a general intellect rendered as fixed algorithmic capital, arguing for a computational form of politics. Parisi, however, points out that in order to repurpose instrumentality, it is imperative, first, to recognise transcendental computation: a transcendental form of reasoning with its own relations between truths and proof, and own forms of knowledge found where once there was only instrumentality, machines enlivened by and in the service of men.

To argue for a transcendental computation, Parisi starts with computation understood as experimental axiomatics. Using Chaitin's work, she writes about computational indeterminacy that reconfigures the relations between truth and proof and opens a new space of reasoning. She then briefly considers Turing's work on computability and inevitability of contingency, before moving to non-deductive logic in the work of Dutch intuitionist mathematician E. J. Brouwer. Turing's incomputables, she argues, have a link to Brouwer's ideal trajectories of logic towards infinity rather than pre-determined limits, opening computation up towards transcendence. Instead of turning incomputables into knowledge, computation generates forms of mediation-instrumental patterning of complexity, which is where a distinct epistemological formation of machine knowledge is sited. To account for the political 
possibilities of this experimental form of subject grounded in transcendental dimensions of computation, Parisi turns to the work of C. S. Peirce. This subject deals with infinities through multilogicality, defying the self-determination of the traditional subject by opening up axioms, premises and truths to dynamic re-articulation. A synthetic mode of inferential reasoning here leads to a third level of abstraction that creates meaning from a complexity of relations, dimensions and practices. Such a multilogical subject exceeds the servo-mechanical neoliberal production of the subject, uniting non-identical scales of causality and transforming reasoning itself.

3. Kolozova's contribution to this special issue is framed as a critique of a certain tradition of thinking, which she broadly labels post-structuralist, and which she, following Laruelle, positions as "irrecuperably Cartesian" in its inescapable division between, on one hand, physicality, materiality and the real, and, on the other, the "phantasmatic" constitution of the self. Here, for Kolozova, the subject is always divorced from the principle of the real and is always bound to remain an ideational construction. Moreover, she argues, the notion of the subject cannot but be modelled on a narrowly perceived human, thus always remaining bound to the anthropological order.

Kolozova's paper offers an argument at a tangent to the positions expressed in the previous two articles: Goriunova's contribution, arguing for a recognition of novel computational forms of establishing relations between subjectivities and the physical, biological principles, which seek to designate reality, truth and value from within their practices, and Parisi's work, arguing for an alien subject of AI, which arises out of new logic and truth procedures of computation. Whereas Goriunova's paper deals with the biological and Parisi's with thought, Kolozova talks about the body and cognition through Marx and Laruelle. It is interesting that in Kolozova's work, the body is postulated as and aligned with the real. Though it is a Laruellian real and is very different to "reality" designated through the work of computation and explored in Goriunova's article, such postulates echo the use of the body for the establishment of the truth of reality in biometrics and other data practices. The problem of the body is something, Kolozova claims, which exhibits the limits of certain traditions of thought. Omnivorous practices of data collection and processing also try to readdress bodies and thoughts, face geometry and photographic conventions, bounding them together in new ways. Kolozova proposes a radical dyad of the non-human self, glued into continuity between the pre-subjective technical automaton of signification and the real of the animal body and the machine as prosthesis moulded together. This non-human dyad never becomes one and never gets unified into truth, - - a radically different result to that aspired to by certain computational practices, seeking to unite, layer and stitch together realities and significations into data analytical patchwork quilts stitched to cover the world. Perhaps such a nonhuman dyad is another proposal for a radical politics today.

Kolozova's proposition is presented at the beginning of her contribution to this special issue. She then proceeds to lay out some of the principles of Laruelle's nonphilosophy, in his critique of philosophy's relation to the real that remains inaccessible to thought and that hence traditionally get cancelled by being converted into "truth". The real nevertheless affects the thinking subject through trauma (Lacan). The human that Kolozova calls the non-human, is a radical dyad of body, machine 
and the automaton of transcendence, of signification, where the automaton itself constitutes a plane of reality. The real then is the exteriority of the signifying chain, and subjectivity is an instance of transcendence, an effect of language. The real (as the body, and the machine) is also within the automaton that produces subjectivisation, but remains outside of it. The self (a wider category than subjectivity) is a dyad of discrete instances of the one ("one of the first names of the real"). In the second part of the article, Kolozova traces a genealogy of the non-unitary subject in critical theory, calling for a restoration of the position of the "one", not as a totalising or reductivist unity, but a new form. To conclude, she turns back to non-relational philosophy of Laruelle to propose instances of oneness for the self, the hybrid of the non-human.

4. Wark takes the notion of the digital subject as a starting point; he then argues that digital subjects keep on individuating in the networks, in a manner that has little to do with human selves. It is the insufficiency and partiality of digital subjects that helps characterise digital subjects as generative of a new mode of subjectivity. It is important then to recognise that what individuates online is not human subjectivity that is extended into the networks, but digital subjects themselves. Digital subjects are both mediate entities and mediators that act as Simondonian "technical entities". These subjects are subjects of circulation (a concept of media that Wark proposes here) that is constitutive of them.

To argue his case, Wark starts by positioning the digital subject vis-à-vis the notion of the user. The user is a construct that expresses the human in computation, and, Wark argues, is employed as a point of differentiation from the technical modes of subjectivity of the digital subject. The meeting point between users and digital subjects is an interface: the users interact on this side of the interface, by providing "representations" of their actions, while the digital subjects is what emerges on the other side of the interface, as digital subjects are aggregated from data by computational services. However, argues Wark, the interfaces are multiple, and the distinction between a user position for the human, a remnant of human computer interaction design, and a user position abstracted further up or down to act in an interface between two programming languages, for instance, is arbitrary. It is then, in his view, multiple user positions constituted in various interfaces that are productive of the digital subjects.

The digital subject is not only about agency sited in such user positions, but a form of individuation, - of itself and of humans. In preparing the conceptual grounds for this claim, Wark uses a critical theory of platforms and Philip Agre's notion of "grammars of action" to analyse Amalia Ulman's project Excellences and Perfections. Here, as programmable platforms scale the operations available to a user position, the constitution of the digital subject enters circulation wherein it becomes technical. To substantiate this claim, Wark turns to the work of Simondon and Hui: digital subjects are technical entities that are ontologically distinct. Concrete operativity, ability to regulate its operations in relation to its environment (associated milieu), is what endows technical entities with their own form of sufficiency. But it is its individuation amidst circulating data that makes the digital subject a subject. Regarding technology as a third mode of being and using a theory of individuation (both Simondon's concepts), in a form re-worked by Yuk Hui, Wark 
argues for a recognition of the digital subject as a technical entity that individuates. In conclusion, Wark returns to Ullman's work and analyses another artist, Zach Blas's work, in terms of a politics of visibility. Wark argues that the drive to be visible positions the digital subject as "the medium" through which platforms" "grammars of action" individuate human beings. People become a core component of the "associated milieu" of the digital subjects, inverting the relation between the digital subject and the human user.

5. Key to Skeggs' and Yuill's contribution is how digital subjects are made in relation to value and values: they follow the line of argument examining the relations between property and propriety and the experience of self-ownership as key to the making of an individual. The conditions of possibility for personhood here are always framed by capitalist forms of value: personhood can be seen as property from which one can derive value. And it is the performance of value, in turn, that is required for the acquisition of modern forms of personhood, confirming the agency of the privileged bourgeois subject.

Digital subjects continue with the same modes of subject-construction: Facebook, the focus of their article, capitalises on people's attributes, aggregating them into digital subjects that are sorted into valuable and valueless. Digital subjects thus continue the property logic of personhood, yet modify it: they lack agency, circulate independently from, and do not return the investment of value to, persons; all value goes to Facebook. Facebook extends and expands the position of the bourgeois, articulate, enterprising subject of value to all its users and demands from them the performance of the "authentic individualised subject of value". The platform then extracts property from the person through curation and aggregation-and does not attribute it: the subjects that expropriate now become subjected to expropriation.

Reporting on their 3-year long research project, Skeggs and Yuill present two women in the complexity of their lives, describing their subject positions, their digital subjects and digital personas. Skeggs and Yuill define digital subjects through the interest that networked platforms, such as Facebook, have in them. As such, these digital subjects are born in relation to and live in the context of a particular social networking platform: they are data assemblages that things are 'being done to". While personhood is changing in relation to the digital infrastructures, old formations and powerful subject positions still have hold over and extend into new spaces, meshing with the new forms. Subject positions are something that can be taken; subjectivity, on the contrary, arises out of work on the self, and the women Skeggs and Yuill describe also perform their subjectivities online. The performance, the telling of a certain subjectivity and not a living of it, is done through the crafting of a digital persona. Curating one's digital persona is perhaps where Facebook's work on the digital subject as data sets is mirrored by the performance of a subjectivity rather than the presence of an authentic personhood. It is in, on one side, the attempts to police and solicit authentic living of a self online rather than a telling of a living, and, on another side, the craftful practices of maintaining personas, that the current lines of tension between techno-capitalist structures and selves lie.

The task of this special issue on digital subjects is to map a field of enquiry that concerns computation and persons. The mapping has made it clear that in order to engage with such a topic, one has to attend to the processes of abstraction and 
modelling, to computational practices in data infrastructures as well as to the processes of subject formation, the questions of power, body, identity, reason and knowledge, reality, art, truth and value. The articles gathered here follow their own paths in investigating subjects produced in and through computation. These are offered as starting points for a larger discussion, which will undoubtedly gather momentum over the next few years.

\section{References}

Althusser, L. 1970. Ideology and ideological state apparatuses (notes towards an investigation). https:// www.marxists.org/reference/archive/althusser/1970/ideology.htm. Accessed 11 Nov 2018.

Anderson, C. 2008. The end of theory. the data deluge makes the scientific method obsolete. The Wired. 06.23.2008. https://www.wired.com/2008/06/pb-theory/. Accessed 11 Nov 2018.

Blackman, L. 2012. Immaterial bodies: Affect, embodiment, mediation. London: Sage.

Braidotti, R. 1994. Nomadic subjects. Embodiment and sexual difference in contemporary feminist theory. New York: Columbia.

Foucault, M. 1982. The subject and power. In Michel Foucault: Beyond structuralism and hermeneutics, ed. H.L. Dreyfus and P. Rabinow. Chicago: The University of Chicago Press.

Foucault, M. 2005. The Hermeneutics of the Subject. Lectures at the College de France 1981-1982. London: Palgrave Macmillan.

Garcia Martinez A. 2018. Ideas. How trump conquered Facebook-without Russian ads. The Wired. 02.23.18. https://www.wired.com/story/how-trump-conquered-facebookwithout-russian-ads/. Accessed 11 Nov 2018.

Goriunova, O. 2019. The digital subject: People as data as persons. Theory, Culture and Society. In print. Guattari, F. 1989. The three ecologies. London: The Athlone Press.

Halpern, O. 2014. Beautiful data: A history of vision and reason since 1945. Durham: Duke University Press.

Haraway, D. 1984. A cyborg manifesto: Science, technology, and socialist-feminism in the late twentieth century. In (1991) Simians, cyborgs and women: The reinvention of nature. London: Routledge.

Kittler, F. 1990. Discourse networks 1800/1900. Stanford: Stanford University Press.

McLuhan, M. 1964. Understanding media: The extensions of man. New-York: McGrow-Hill.

Moulier-Boutang, Y. 2012. Cognitive capitalism. London: Polity.

Rouvroy, A. 2013. The end(s) of critique: Data behaviourism versus due process. In Privacy, due process and the computational turn, eds. M. Hildebrandt and K. de Vries. London, New York: Routledge.

Stiegler, B. 1998. Technics and time, 1: The fault of Epimetheus. Stanford: Stanford University Press.

Valiaho, P. 2014. Biopolitical screens: Image, power and the neoliberal brain. Cambridge: MIT Press.

Olga Goriunova is Reader and Director of Research at the Department of Media Arts, Royal Holloway University of London. Her forthcoming book is Bleak Joys. Aesthetics of Ecology and Impossibility (coauthored with Matthew Fuller; University of Minnesota Press, 2019). She is the author of Art Platforms and Cultural Production on the Internet (Routledge, 2012), editor of Fun and Software: Exploring Pleasure, Pain and Paradox in Computing (Bloomsbury, 2014) and co-editor, with Alexei Shulgin, of Readme. Software Art and Cultures (University of Aarhus Press, 2004). She is a co-founder and co-editor of Computational Culture, A Journal of Software Studies (computationalculture.net). 